\title{
Influence of an Aperture on the Performance of a Two-Degree-of-Freedom Iron-Cored Spherical Permanent-Magnet Actuator
}

\author{
J. K. Mitchell, G. W. Jewell, J. Wang, C. M. Bingham, and D. Howe
}

\begin{abstract}
This paper describes a computational and experimental study of a two-degree-of-freedom spherical permanent-magnet actuator equipped with an iron stator. In particular, it considers the effect of introducing an aperture in the stator core to facilitate access to the armature. The resultant magnetic field distribution in the region occupied by the stator windings, the net unbalanced radial force, and the resulting reluctance torque are determined by three-dimensional magnetostatic finite-element analysis. The predicted reluctance torque is validated experimentally, and its implications on actuator performance are described.
\end{abstract}

Index Terms-Finite-element modeling, permanent-magnet actuators, reluctance torque, spherical actuators, unbalanced magnetic pull.

\section{INTRODUCTION}

$\mathbf{S}$ PHERICAL permanent-magnet actuators, capable of producing motion with either two or three degrees of freedom (DOF), are being developed for applications that require precise and complex motion control, such as stereoscopic active vision systems and force feedback joysticks. To date, however, only spherical actuators employing air-cored stators or stators in which the windings were contained within a smooth, infinitely permeable spherical iron core have been considered [1]-[6]. Such spherical actuators comprise an appropriately magnetized permanent-magnet rotor around which are disposed a number of stator coils and linear Hall sensors for rotor position sensing. Excellent closed-loop servo-characteristics have been demonstrated, albeit the actuators having only moderate specific torque capabilities [3].

The use of an iron-cored stator, either slotless in which the coils are surrounded by a smooth spherical iron shell or slotted in which the coils are wound around a toothed iron core, can considerably enhance the specific torque capability. For example, previous analysis, has demonstrated that the torque capability of a slotless iron-cored two-DOF actuator would be some $30 \%$ higher than for a similarly sized air-cored actuator [3]. This analysis was, however, based on a simplified model of the actuator, which assumed that the spherical iron core completely surrounded the permanent-magnet rotor and stator coils, and, hence, maintained the rotational symmetry so as to enable an exact analytical field solution to be derived.

Manuscript received November 27, 2001; revised August 6, 2002. This work was supported by the U.K. Engineering and Physical Sciences Research Council under the Electrical Machines and Drives Program.

The authors are with the Department of Electronic and Electrical Engineering, The University of Sheffield, Sheffield S1 3JD, U.K.

Digital Object Identifier 10.1109/TMAG.2002.804812
However, in a practical iron-cored spherical actuator, it would be necessary to incorporate at least one aperture in the iron core in order to gain access to the rotor so as to attach the external payload, e.g., a camera in an active vision system, the handle of a force-feedback joystick, etc. Such an aperture would modify the magnetic field distribution, which, in turn, may degrade the performance of the actuator in three respects, since it may result in the following:

1) an asymmetrical flux density distribution, which would affect the excitation torque versus rotor angular displacement characteristics;

2) a reluctance torque component, due to the interaction of the permanent-magnet field and the asymmetrical stator iron geometry;

3) asymmetry in the radial force distribution and, hence, an "unbalanced magnetic pull," which could impose a significant additional load on the bearing system.

This paper describes a computational and experimental investigation of the influence of an aperture on the performance of a slotless iron-cored two-DOF permanent-magnet spherical actuator.

\section{ACTUATOR SPECIFICATIONS}

The investigation is based on the iron-cored two-DOF spherical actuator whose leading dimensions and stator geometry are shown in Fig. 1. The permanent-magnet rotor is a 48-mm-diameter sintered anisotropic $\mathrm{Nd}-\mathrm{Fe}-\mathrm{B}$ sphere (UGIMAG $37 \mathrm{~B}$; with a remanence of $1.28 \mathrm{~T}$ ) which is magnetized diametrically. It is the same rotor as that which was employed previously in an air-cored two-DOF spherical actuator which had torque capabilities of $0.15 \mathrm{Nm}$ (continuous) and $0.4 \mathrm{Nm}$ (peak) [3].

A 13-mm-diameter flat with a 5-mm-deep, 5-mm-diameter hole was machined in the rotor in order to facilitate the attachment of the aluminum payload arm. The 88-mm inner diameter of the stator iron core, accords with the optimal "split-ratio" for maximizing the specific torque capability, as described in [1], [3], while the aperture was dimensioned to accommodate $\mathrm{a} \pm 45^{\circ}$ pan and tilt excursion with a $10-\mathrm{mm}$-diameter payload arm. Hence, its inner surface subtends an angle of $\pm 51.5^{\circ}$. The outer diameter of the stator iron core was dimensioned to avoid undue saturation.

\section{Finite-Element Modeling}

Although the actuator has a relatively simple geometry, recourse to three-dimensional (3-D) magnetostatic field analysis is 


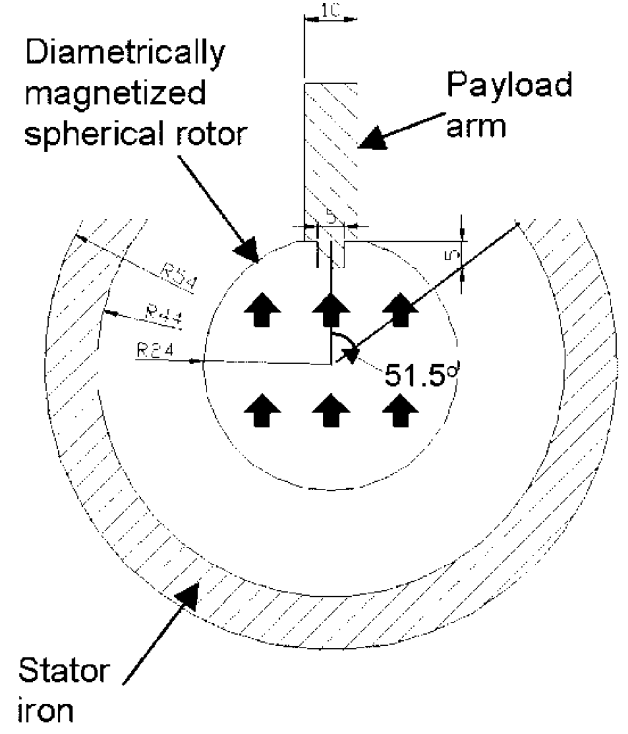

(a)

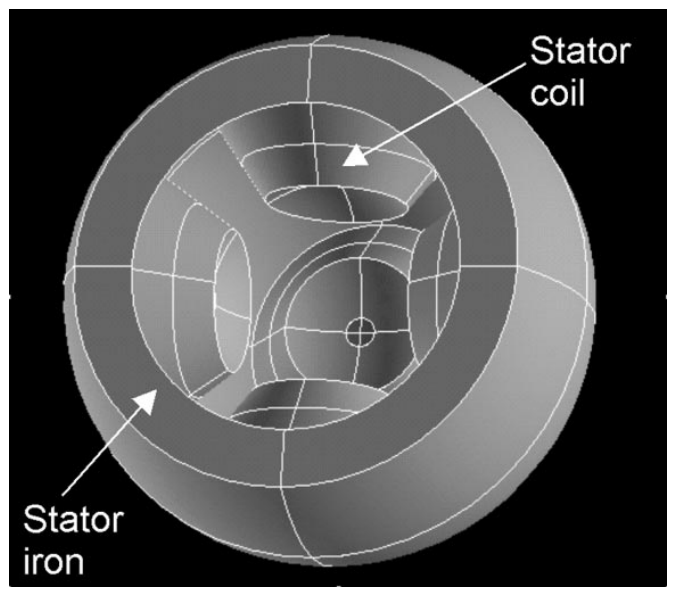

(b)

Fig. 1. (a) Leading dimensions of actuator (in millimeters). (b) Stator geometry (shown with permanent-magnet rotor removed).

necessary in order to determine the magnetic field as the rotor orientation is varied. The actuator was modeled using 3-D finite-element analysis (ANSYS Version 5.6). In order to minimize the computational effort, the stator iron was assumed to have a constant relative permeability of 7000 . As will be demonstrated, this assumption is justified by the fact that the maximum flux density within the iron (which occurs near the corner adjacent to the aperture) is $1.1 \mathrm{~T}$. Thus, the working points lie on the linear region of the magnetization characteristic.

The geometrical symmetry of the actuator was exploited by reducing the problem domain to one half of the actuator, illustrated by the typical 3-D finite-element mesh in Fig. 2, which, in this case, models the actuator for zero rotor displacement. Although the discretization varied with rotor displacement, the number of finite elements was typically in the region of 120000 . In order to ensure that the reluctance force was calculated to an acceptable accuracy, the air space in the vicinity of the aperture was finely discretized, and a 1-mm-thick layer of uniformly discretized elements being employed adjacent to the surface of the rotor. The problem domain was bounded by a tangential flux

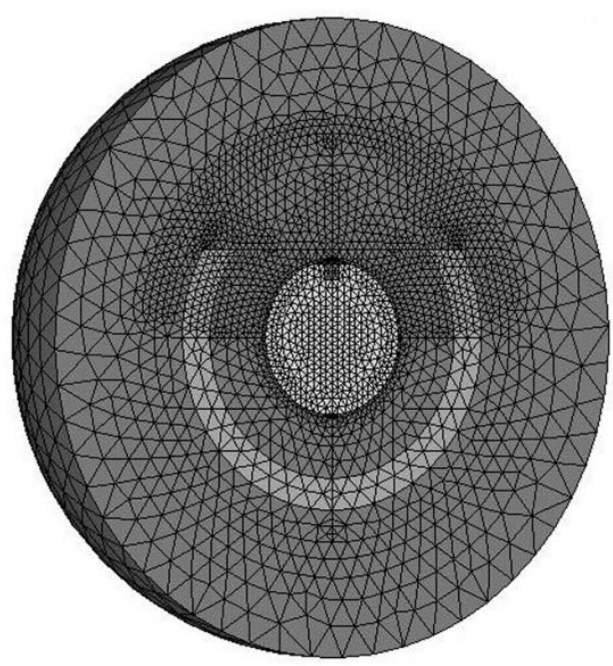

Fig. 2. Three-dimensional finite-element mesh with zero rotor displacement.

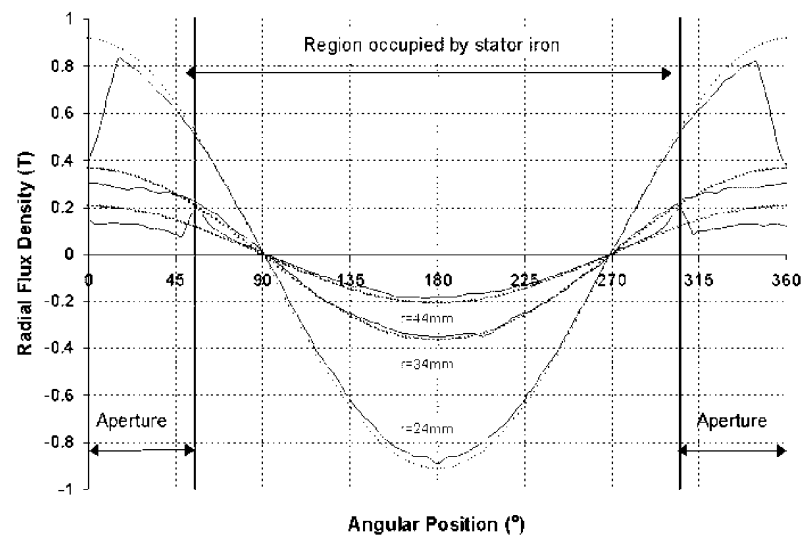

Fig. 3. Predicted radial flux density profiles for zero rotor displacement

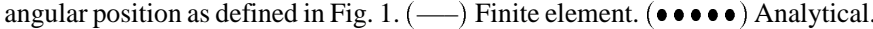

condition imposed at an enclosing spherical boundary having a radius of $100 \mathrm{~mm}$. The open-circuit magnetic field distributions (i.e., with stator coils unexcited) were determined using a scalar potential formulation and employing first-order finite elements (type SOLID96 [7]). For a given angular displacement of the rotor, the reluctance torque and the net unbalanced force were calculated using the Virtual Work method.

\section{PRedicted And Measured Results}

Fig. 3 compares finite-element predicted profiles of the radial component of flux density around three circular paths having different radii, viz. $24 \mathrm{~mm}$ (on surface of permanent magnet); $34 \mathrm{~mm}$ (center of the air gap); and $44 \mathrm{~mm}$ (on inner surface of iron) for zero displacement. Also shown in Fig. 3 are the corresponding flux density profiles calculated analytically assuming that the stator core comprises a complete spherical iron shell [3].

As will be seen, while there is reasonable agreement between the predicted flux density profiles within the region that is enclosed by the stator core, there is a considerable difference between the flux density profiles in the region under the aperture, particularly at the surface of the rotor, where the influence of the payload arm mounting hole is apparent at the stator bore immediately adjacent to the aperture. Similar behavior is observed 


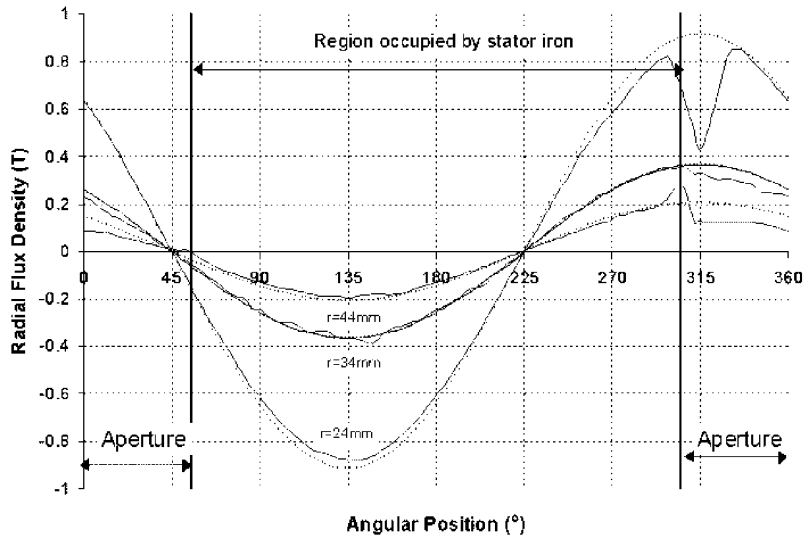

Fig. 4. Predicted radial flux density profiles for a rotor displaced by $45^{\circ}$ angular position as defined in Fig. 1. (-) Finite element. Analytical.

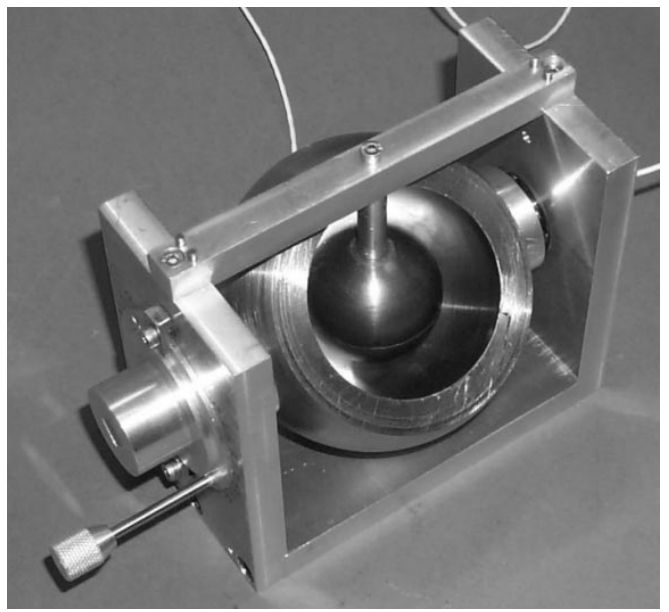

Fig. 5. Experimental test rig for measurement of reluctance torque.

when the rotor is displaced by $45^{\circ}$, as shown in Fig. 4. The excitation torque that results when a particular stator coil is excited is obtained from a volume integration of the radial component of flux density over the volume occupied by the coil. Hence, given that the stator coils are all located entirely within the stator iron core, it is evident from Figs. 3 and 4 that an aperture having the dimensions shown in Fig. 1 will have a negligible influence on the excitation torque characteristics predicted by the analytical design technique described in [3].

The variation of the reluctance torque with rotor angular displacement was calculated from ten finite-element field solutions with increments of $5^{\circ}$ in angular displacement. In order to validate the calculation, the reluctance torque was measured using the test rig shown in Fig. 5, in which the spherical permanent-magnet sphere rotor was rigidly fixed to the base, and the spherical iron stator core was supported by a pair of rolling element bearings. At any given rotor angular displacement (which is set by a precision dowel arrangement), the net reluctance torque was measured by a pair of rotary load cells (ENTRAN ELT-1 M-2.5 NM). The predicted and measured reluctance torque characteristics are compared in Fig. 6, the maximum difference being some $10 \%$ of the peak magnitude.

An interesting feature of the reluctance torque characteristics of Fig. 6 is that they exhibit "negative" stiffness, i.e., at zero

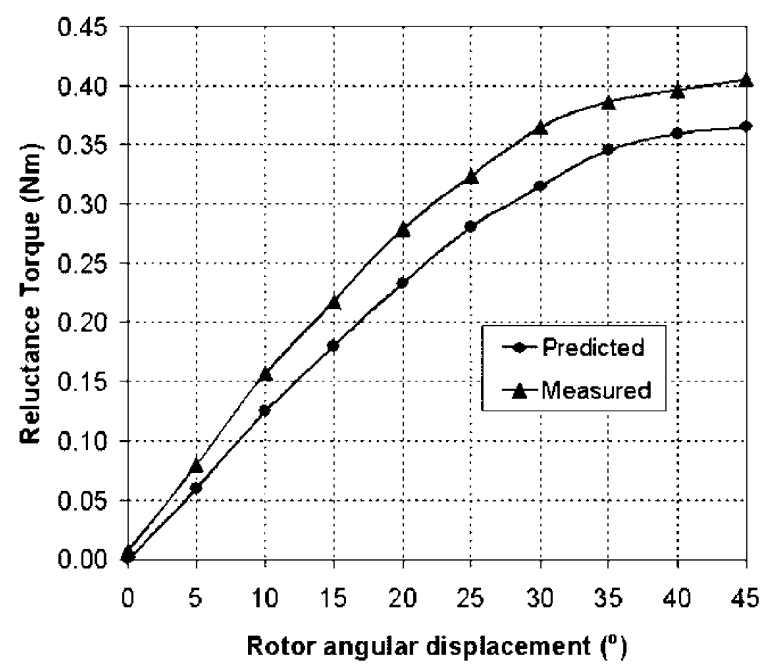

Fig. 6. Comparison of predicted and measured reluctance torque as a function of rotor angular displacement.

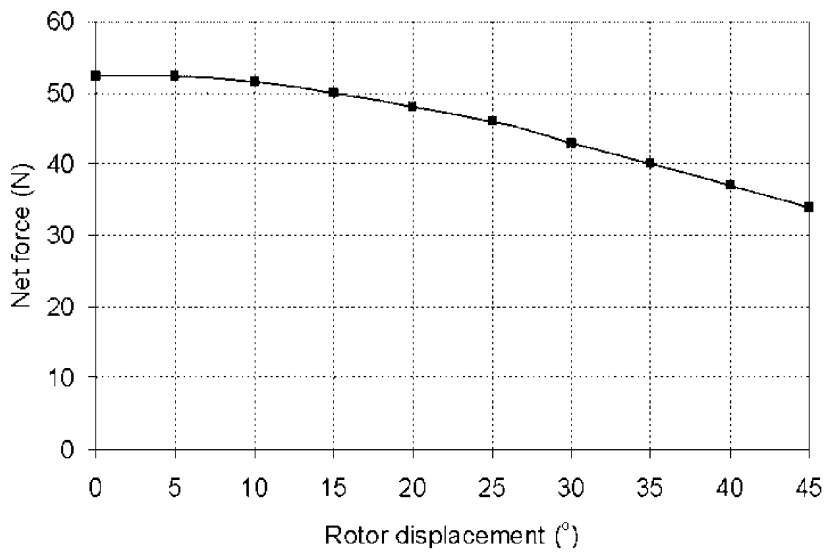

Fig. 7. Predicted magnitude of net radial force as a function of rotor angular displacement.

displacement the rotor is in an unstable equilibrium, and if displaced would rotate to a position of minimum reluctance. For many applications, which require rapid and precise movement of a payload, this is likely to be an undesirable feature, in that it will inevitably compromise the achievable closed-loop dynamic performance. For applications with more subjective performance criteria, such as force-feedback joysticks, it is likely to compromise the haptic "feel" of the device. However, of particular concern is that the peak reluctance torque is comparable to the peak excitation torque rating of $0.56 \mathrm{Nm} \mathrm{[3]} \mathrm{(predicted}$ analytically for a current density of $4 \mathrm{~A} / \mathrm{mm}^{2}$ and no aperture). However, the problem could be largely overcome by incorporating a restoring spring mechanism in the actuator, although it would inevitably be rather complex in order to provide the required restoring forces in the two DOF.

The magnitude and resultant direction of the "unbalanced magnetic pull" that is produced as the rotor is displaced from its equilibrium zero displacement position was also deduced from the finite-element field solutions. The resulting characteristics are shown in Figs. 7 and 8. As can be seen, the magnitude of this force is very significant, being some 12 times the gravitational force exerted by the permanent-magnet rotor and payload arm. 


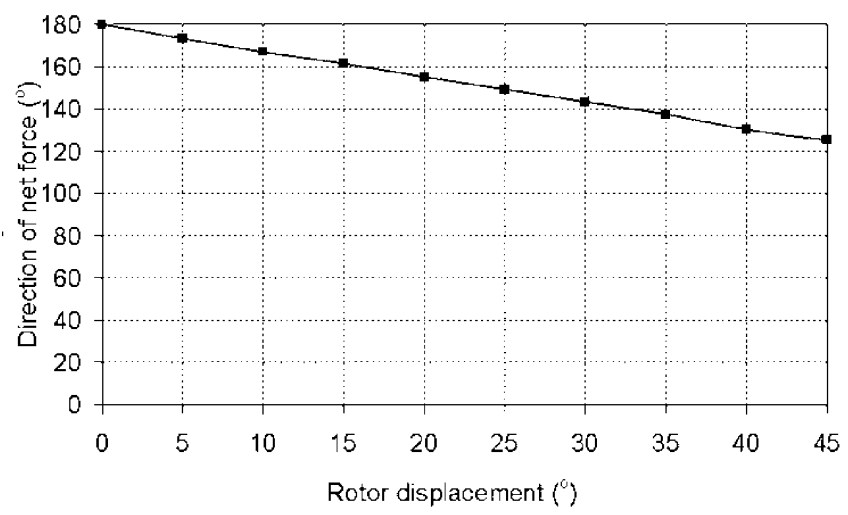

Fig. 8. Predicted direction of net radial force (relative to reference angle defined in Fig. 1) as a function of rotor angular displacement.

In terms of realizing a practical iron-cored spherical actuator, in order to accommodate this magnitude of unbalanced force a relatively complex precision bearing arrangement is likely to be required.

\section{CONCLUSION}

Finite-element analyses and experimental measurements presented have enabled the influence of an aperture in an iron-cored stator of a spherical two-DOF actuator to be quantified. For the particular actuator dimensions considered in this paper, the excitation torque characteristic is not modified to any great extent by the presence of the aperture. However, the presence of the aperture does introduce significant problems in terms of the resulting reluctance torque and unbalanced radial forces. Thus, while an iron-cored stator would increase the volumetric torque capability, it would inevitably required a more complex rotor mechanical support arrangement and, thereby, compromise one of the attractive features of spherical actuators, viz. their relative simplicity. Further, the use of a solid iron stator may compromise the dynamic performance due to the influence of induced eddy currents, although this has not been considered in this paper.

\section{ACKNOWLEDGMENT}

The authors are particularly grateful to J. Morrison and I. Lyne for the design and manufacture of the test rig.

\section{REFERENCES}

[1] J. Wang, G. W. Jewell, and D. Howe, "A novel spherical actuator: Design and control," IEEE Trans. Magn., vol. 33, pp. 4209-4211, Sept. 1997.

[2] — - "Analysis of a spherical permanent magnet actuator," J. Appl. Phys., vol. 81, no. 8, pp. 4266-4268, 1997.

[3] - "Analysis, design and control of a novel spherical permanent magnet actuator," Proc. Inst. Elec. Eng.—Electric Power Applicat., vol. 145, no. 1, pp. 61-71, 1998.

[4] J. Wang, W. Wang, G. W. Jewell, and D. Howe, "A novel spherical permanent magnet actuator with three degrees-of-freedom," IEEE Trans. Magn., vol. 34, pp. 2078-2080, July 1998.

[5] W. Wang, J. Wang, G. W. Jewell, and D. Howe, "Analysis, design and control of a novel spherical permanent magnet actuator with three degrees-of-freedom," IEEE/ASME Trans. Mechatron., submitted for publication.

[6] J. Wang, G. W. Jewell, and D. Howe, "Modeling of a novel spherical permanent magnet actuator," in Proc. IEEE Int. Conf. Robotics and Automation, Albuquerque, NM, Apr. 1997, pp. 1190-1196.

[7] Ansys User's Manual, vol. 3 (Elements)—Section 4.96, Ansys Inc., Canonsburg, PA. 